\title{
Médiévales
}

Langues, Textes, Histoire

48 | printemps 2005

Princes et princesses à la fin du Moyen Âge

\section{Le prince et l'histoire dans le comté de Holstein, au miroir du Chronicon Holtzatiae Auctore Presbytero \\ Bremensi}

Prince and History in the Mirror of the Chronicon Holtzatiae Auctore Presbytero

Bremensi.

\section{Mathieu Olivier}

\section{OpenEdition}

Journals

Édition électronique

URL : https://journals.openedition.org/medievales/833

DOI : 10.4000/medievales.833

ISSN : 1777-5892

Éditeur

Presses universitaires de Vincennes

Édition imprimée

Date de publication : 1 juin 2005

Pagination : 99-122

ISBN : 2-84292-169-0

ISSN : 0751-2708

\section{Référence électronique}

Mathieu Olivier, «Le prince et l'histoire dans le comté de Holstein, au miroir du Chronicon Holtzatiae Auctore Presbytero Bremensi », Médiévales [En ligne], 48 I printemps 2005, mis en ligne le 02 mars 2007, consulté le 24 avril 2022. URL : http://journals.openedition.org/medievales/833 ; DOI : https://doi.org/ $10.4000 /$ medievales.833

Ce document a été généré automatiquement le 24 avril 2022

Tous droits réservés 


\section{Le prince et l'histoire dans le comté de Holstein, au miroir du Chronicon Holtzatiae Auctore Presbytero Bremensi}

Prince and History in the Mirror of the Chronicon Holtzatiae Auctore Presbytero Bremensi.

\section{Mathieu Olivier}

1 Il est un fait que Bernard Guenée a établi dans son ouvrage de référence Histoire et Culture historique dans l'Occident médiéval: le $\mathrm{xv}^{\mathrm{e}}$ siècle voit proliférer les histoires qui sont des « écrits politiques rédigés à une certaine date pour une certaine cause ${ }^{1}$. C'est cette idée-force qui nous a servi de fil conducteur pour relire un texte bien oublié du $\mathrm{xv}^{\mathrm{e}}$ siècle allemand ${ }^{2}$.

2 Non pas que le Chronicon Holtzatiae auctore Presbytero Bremensi ait été complètement négligé au sortir du Moyen Âge. Cette chronique universelle, mais centrée sur la terre de Holstein - «terra Holsacie » - et écrite en 1448 par un prêtre anonyme originaire de la région, a été éditée trois fois à l'époque moderne. L'édition princeps est due au grand Leibniz lui-même en 1698. Suivront deux autres éditions, l'une par l'érudit Westphalen dans ses volumineux Monumenta Inedita ${ }^{3}$, l'autre en 1862 par Lappenberg ${ }^{4}$. Il n'en reste pas moins que le Presbyter Bremensis n'a guère été tenu en haute estime: latin désastreux, imagination fertile, passages abscons, les critiques ont plu à l'époque de l'histoire positiviste sur un texte qui semblait avoir pour seul mérite de pallier l'absence d'œuvre historique de grande envergure prenant le relais d'Adam de Brême et d'Helmold de Bosau pour les derniers siècles du Moyen Âge. J'aimerais essayer de montrer ici, en quelques pages qui synthétisent les résultats et les conclusions d'un travail de maîtrise ${ }^{5}$, que l'on gagne incontestablement à considérer le Chronicon à la lumière d'une approche différente : que nous dit ce texte du milieu du xve siècle sur la conscience de soi de la dynastie des comtes de Schauenburg qui détient le comté de Holstein en fief depuis 1111 ? Que peut-il nous apprendre de la construction d'une identité régionale, processus que l'on voit à l'œuvre, à cette époque, dans bien d'autres 
territoires du Saint Empire? Telles sont les interrogations auxquelles je vais tenter ici d'esquisser des réponses.

L'auteur et son œuvre

3 Mais qui est pour commencer ce mystérieux Presbyter Bremensis et, pour reprendre la terminologie de Bernard Guenée, a-t-il écrit pour une cause politique particulière ? Son œuvre en tout état de cause dénote une très faible familiarité avec les textes historiques antérieurs. Sa source principale et sa référence - puisqu'il s'en dit lui-même le « continuateur » - est Helmold de Bosau et sa Cronica Slavorum. Mais au-delà de 1170, son récit ne semble tributaire d'aucune œuvre narrative importante. Cette particularité est aussi une opportunité inestimable : notre auteur est, par là même, moins prisonnier que certains de ses contemporains de schémas historiographiques anciens; sa relation nous livre ainsi, sans qu'aucun système historiographique ne vienne faire écran, une formulation de l'Histoire des régions au nord de l'Elbe - autrement dit «nordalbingiennes" pour reprendre un mot médiéval, bien commode en l'occurrence-, plus que jamais ancrée dans les réalités politiques, dynastiques mais aussi conceptuelles de son temps. Notre auteur se définit lui-même comme scriba hujus patrie $^{6}$ : nous avons très vraisemblablement affaire ici à un clerc de modeste rang officiant dans une " chancellerie » comtale encore largement embryonnaire ; l'examen des actes (peu nombreux) qu'il paraît avoir utilisés pour la rédaction de sa chronique nous conduit à penser que, s'il n'avait sans doute qu'une connaissance " accidentelle " des documents comtaux, il semble vraisemblable qu'il ait participé directement aux négociations de 1447 à Lubeck entre le duc de Schleswig et comte de Holstein Adolphe VIII et les représentants de ses turbulents voisins occidentaux, les paysans de facto libres des Dithmarschen, région de polders coincée entre le Holstein et le Mer du Nord : soit très peu de temps avant qu'il n'entreprenne de rédiger sa chronique latine.

Par ailleurs, il nous est loisible d'inférer du Chronicon un certain nombre d'hypothèses concernant l'environnement du Presbyter, et ce plus précisément que ne l'avaient fait Westphalen ou Lappenberg. Son affection particulière pour Itzehoe, corroborée par son insistance sur les régions occidentales du Holstein (Ditmarschen, Kremper et Wilstrer Marschen) indique qu'il était probablement originaire de cette région, et sa particulière acribie pour tout ce qui concerne les inhumations comtales à Itzehoe, ainsi que la précision de ses informations concernant la vie locale, paraissent signaler qu'il entretenait des liens étroits avec le couvent des cisterciennes de ce même bourg, liens pressentis déjà par Lappenberg. Or Itzehoe jouait alors à l'égard du lignage Schauenburg le rôle d'authentique "monastère dynastique »: depuis le xIV siècle la plupart des dynastes y furent enterrés, et, à partir de 1421, l'entretien minutieux de la memoria comtale, par des messes commémoratives et d'autres obligations cultuelles, est attesté. Nous avons de fait de bonnes raisons de penser que ce Presbyter Bremensis fut attaché, d'une façon ou d'une autre, au couvent d'Itzehoe, peut-être parmi les quelque vingt vicaires attachés à l'église Saint-Laurent/Notre Dame d'Itzehoe, en tout cas au sein du pléthorique clergé masculin qui gravitait autour de la nécropole comtale $^{8}$.

Une dernière pièce du puzzle nous manque encore pour approcher de plus près les motivations réelles du Presbyter Bremensis, les ressorts et les enjeux sous-jacents du Chronicon Holtzatiae: le contexte politique au nord de l'Elbe dans ce qui allait être les dernières années de la mainmise des Schauenburg-Rendsburg sur le comté de Holstein. Une crise dynastique se profile à l'horizon. Le comte Adolphe VIII, dernier des 
Schauenburg-Rendsburg, n'a pas d'héritier, et le futur du comté - mais aussi du duché de Schleswig, ancien apanage stratégique de la couronne danoise progressivement arraché à la domination de celle-ci depuis le XIv ${ }^{e}$ siècle, et objet de rivalités jamais éteintes entre les comtes et les rois danois ${ }^{9}$ - est incertain. Deux prétendants sont en lice: d'un côté les cousins de la branche SchauenburgPinneberg, possessionnés essentiellement dans la vallée de la Weser, berceau de la dynastie, et de l'autre Christian d'oldenburg, qui a épousé la sœur d'Adolphe VIII en 1421. Ce dernier prétendant devient par ailleurs, assez inopinément, roi du Danemark, précisément en 1448 ; il est soutenu par Adolphe VIII, au détriment de ses cousins Schauenburg, et en dépit du droit féodal qui ne reconnaît pas la succession du fief par les femmes. Quand on sait que le Chronicon présente à la fois une tendance nette au dénigrement des Pinneberg, et n'a de cesse de rappeler les liens des comtes avec le centre d'Itzehoe, le mystère s'éclaircit. Le Presbyter a peut-être écrit " pour compléter la chronique fidèle des princes des Holsten et de leurs voisins que frère Helmold de pieuse mémoire avait composée $\aleph^{10}$, mais son objectif premier était loin de l'émulation désintéressée : il s'agissait sans doute à la fois d'appuyer les préférences successorales du dernier des Schauenburg-Rendsburg et, à la veille d'une rupture dynastique qui était synonyme de remise en cause des situations acquises et des privilèges anciens liés à la célébration de la memoria des Princes, de prendre des assurances pour l'avenir en incitant le nouveau dynaste à pérenniser la fonction de nécropole d'Itzehoe. Il nous paraît indubitable que nous avons là affaire à un texte nettement " conjoncturel », beaucoup plus en tout cas que semblaient le croire ses éditeurs.

La « terra » et le Prince : l'image d'un lien consubstantiel

6 Cela dit, cet ancrage dans une conjoncture politique bien déterminée ne signifie pas qu'il faille d'emblée y chercher uniquement ce que l'on est en droit d'attendre d'un "texte de circonstance ». À la lumière de cette situation historique particulière, examinons plutôt ce qu'il peut nous apprendre de l'articulation entre conscience dynastique et identité de la terra Holtzacie. Un fait s'impose : la conscience que l'on pourrait dire proprement lignagère n'a pratiquement aucune consistance sous la plume du Presbyter Bremensis. Rares sont les excursus généalogiques, où l'Histoire de la terra disparaît derrière l'évocation de la domus Schauenburgensis. Cette notion même de domus, si cruciale à la même époque pour la compréhension de l'identité princière de grands lignages de l'Empire comme les Wittelsbach minutieusement étudiés par JeanMarie Moeglin ${ }^{11}$, est quasiment absente du Chronicon, à l'exception d'occurrences isolées pour désigner la «Maison de France $»^{12}$ ou la «Maison de Brunswick $»^{13}$ (en réalité la famille des Welfs); l'intérêt qu'a notre auteur à minorer les solidarités du sang à l'égard de la branche Pinneberg joue certainement un rôle, mais il semble parallèlement que le concept de domus soit dans l'esprit du Presbyter associé aux seuls grands lignages d'Empire et aux familles royales ou princières étrangères, même s'il faut noter que le Presbyter l'emploie malgré tout une fois pour désigner la Maison de Schauenburg ${ }^{14}$. La fascination pour le titre ducal qui se fait jour dans notre chronique marque peut-être le pendant de cette dure réalité dont le Presbyter se fait l'écho en filigrane : les Schauenburg ne cessent d'aspirer à un rang disons "princier »-au sens des « Princes d'Empire » - qui, malgré tous leurs efforts, reste encore bien éloigné. Le titre de duc de Schleswig, bien que concernant une terre hors de l'Empire, joue manifestement un rôle non négligeable dans le processus d'affirmation des Schauenburg au $\mathrm{Xv}^{\mathrm{e}}$ siècle : aux titulatures tronquées où seul le titre ducal apparaît, fréquentes dans les actes comtaux au $\mathrm{Xv}^{\mathrm{e}}$ siècle ${ }^{15}$, répond l'ire de notre auteur devant le 
supposé déni de titre perpétré par le roi du Danemark Éric de Poméranie au temps du paroxysme des tensions autour de la domination du duché de Schleswig: «contre les princes de Schleswig et comtes de Holstein, en ne les appelant dans ses lettres que comtes et non ducs $»^{16}$.

Il n'en reste pas moins que la relation articule continûment destin de la dynastie et destin de la «terre de Holstein ». L'une ne peut se concevoir sans l'autre, tel semble être l'axiome de ce discours de la conscience princière. La numérotation dynastique appliquée par notre auteur amène, significativement, à faire de la date de 1111, inféodation des Schauenburg pour le comté de Holstein, l'acte de naissance de la famille, au rebours du récit d'Hermann de Lerbeck, enclin de son côté à démontrer que les Schauenburg existaient dans la vallée de la Weser bien avant cette date - ce qui, à la lumière de l'état actuel de la recherche semble probable, bien que très mal attesté ${ }^{17}$. On notera par ailleurs le silence total du Presbyter Bremensis sur les épisodes bien réels de rupture de la continuité dynastique dans le comté de Holstein entre 1111 et 1448 : deux « usurpations » éphémères, en 1137-1138 et en 1148-1149, auxquelles le récit d'Helmold de Bosau fait pourtant explicitement référence, disparaissent purement et simplement dans le Chronicon. Plus révélatrice encore de cette tendance de fond est l'élimination de la figure de l'«anti-comte » Albert d'Orlamünde : ce personnage, installé en 1201 par les Danois qui viennent de chasser de ses possessions nordalbingiennes le comte Schauenburg Adolphe III, se maintient jusqu'en 1227 quand il est chassé par le jeune Adolphe IV reconquérant le comté après la victoire de Bornhöved. Alors que tout un pan de l'historiographie régionale depuis le xiIl siècle intègre, sans réticence particulière semble-t-il, cette rupture ${ }^{18}$, celle-ci est complètement escamotée dans le Chronicon : «Il n'y avait pas alors de prince dans la terre de Holstein » (Illis diebus non erat princeps in terra Holtzacie) se borne-t-il à constater après avoir rapidement évoqué les revers militaires des Schauenburg en 1200-1201, et avant de revenir sur le triomphe de Bornhöved. Il paraît difficile de croire que notre auteur ait ignoré de bonne foi cet épisode. Continuité dynastique sans faille et pérennité de la terra vont donc de pair ici et la proximité de notre auteur avec le cercle d'Adolphe VIII nous incline à voir en lui, conjointement, un porte-parole et un artisan à part entière d'une identité dynastique fermement arrimée à la terre de Holstein.

8 En prise avec son temps, la conscience dynastique qui se fait jour à travers notre chronique l'est aussi sur un autre plan. Les Schauenburg semblent soucieux de se doter d'une origo prestigieuse. Et, tout à fait en accord en cela avec les canons du xve siècle, ils la veulent antique et romaine. Elle plonge même doublement dans le monde romain, à en croire le récit intéressant du Presbyter : en premier lieu, la construction du Château de Schaumburg, berceau de la dynastie, remonterait à Jules César en personne ; et, par ailleurs, les Schauenburg seraient apparentés à la famille romaine des Orsini ${ }^{19}$, peutêtre sur le modèle contemporain des ducs du Mecklembourg voisin, qui eux aussi prétendaient descendre de cette illustre famille. L'originalité du récit du Presbyter à cet égard est ailleurs. Tout d'abord, l'avènement relativement tardif de la dynastie dans le comté de Holstein paraît poser problème à notre auteur, en ce qu'il répugne visiblement à pleinement faire exister "politiquement» la terra avant l'arrivée des Schauenburg, au début du XII ${ }^{\mathrm{e}}$ siècle. Il supprime ainsi les rares mentions de comites qui paraissent s'appliquer à l'espace au nord de l'Elbe dans le texte d'Helmold, tout comme il éclipse un mystérieux «comte Bernard » figurant dans la "Vita Anskarii » ${ }^{20}$, dont le Presbyter a manifestement utilisé une version pour sa chronique. Tout comme les 
Schauenburg ne peuvent avoir vraiment eu une existence avant leur arrivée dans le Holstein, la terra ne peut avoir eu une authentique réalité politique avant les Schauenburg.

9 Mais le récit du Presbyter présente une autre facette singulière de l'articulation entre origo dynastique et terre de Holstein: il procède pour ainsi dire à la mise en place historiographique d'une deuxième origo. Celle-ci est rendue nécessaire par un phénomène essentiel de l'histoire du comté du dernier tiers du xiII siècle à la fin du XIV siècle, à savoir les multiples partages territoriaux auxquels se livrent des dynastes Schauenburg qui ignorent la pratique de la primogéniture. Cette étape historique n'est close qu'en 1390, lorsque la branche Rendsburg réunifie à son profit la totalité du comté, à l'exception des maigres possessions de la branche Pinneberg autour de Hambourg. Si le Presbyter avalise sans scrupules apparents le principe de divisibilité de la terra, il s'attache à faire de l'ascension des Schauenburg-Rendsburg - brutale et émaillée de dénis de droits et de fratricides dans la réalité - l'histoire édifiante de la revanche patiente d'une branche cadette injustement spoliée aux temps des premiers partages contre des cousins tyranniques, cupides et dégénérés.

10 À un éparpillement territorial fait d'enclaves et de discontinuités, le Presbyter substitue plus ou moins explicitement, sans qu'il soit vraiment possible de faire ici le départ entre l'ignorance et la mauvaise foi, un schéma simpliste et « idéologiquement » commode pour asseoir la légitimité des descendants des Schauenburg-Rendsburg qui règnent sans partage sur le comté jusqu'à 1459 : les Teilgrafen Rendsburg détiennent l'ouest de la région nordalbingienne, y compris la Holtzacia proprement dite, alors que les autres branches sont concentrées à l'est, autour de Lubeck, territoire anciennement slave et intégré beaucoup plus tardivement au cœur du comté. Il est permis de penser que cette dichotomie n'est pas innocente en termes de légitimité politique, si l'on se penche sur les conséquences ultimes de ce schéma: les "comtes de l'Est», ceux auxquels s'opposent les Rendsburg dans leur reconquête du comté, sont parfois appelés seulement « comtes de Wagrie » et non plus comtes de Holstein ${ }^{21}$. On peut se demander s'il n'y a pas là comme une façon de justifier la lutte des Rendsburg pour les exclure de la domination sur le comté.

11 Les grandes victimes de cette réécriture de l'histoire sont, comme nous le suggérions d'entrée, les cousins de la branche Pinneberg. Leur ancrage territorial au nord de l'Elbe, qui se fixe précisément à l'ère des partages territoriaux, n'est jamais décrit dans la géographie des Teilgrafschaften que tente de brosser le Presbyter. Leurs interventions au nord de l'Elbe, destinées bien souvent à contrer la politique hégémonique des Rendsburg, sont présentées comme d'infamantes opérations de représailles montées pour venger la mort de parents indignes ${ }^{22}$. Là encore est perceptible une propension discrète à n'accorder aux Pinneberg qu'une titulature arbitrairement tronquée: "comte de Schauenburg " ${ }^{23}$. L'évocation du traité de "réunification» du comté en 1390, destiné précisément à fixer les règles d'un modus vivendi acceptable entre les deux branches survivantes (Rendsburg et Pinneberg), nous permet par bonheur de comparer l'explicitation qu'en fait le Presbyter et la lettre du texte qui nous est parvenu ${ }^{24}$. Alors que le traité en question stipule toute une série d'obligations destinées à maintenir une solidarité minimale, sinon une effective cogestion du comté, entre les deux branches, le Presbyter passe outre et se contente alors de rapporter: "le comte Nicolas, qui lui survivait, réunifia sous son autorité toute la terre de Holstein» («Comes Nicolaus superstes [...] reduxit totam terre [sic !] Holtzacie in unum dominium »). Ignorance ou silence 
complaisant, peu importe finalement: dans l'entourage d'Adolphe VIII de Schauenburg-Rendsburg, et alors que la crise dynastique se profile à l'horizon, on se montre manifestement peu enclin à mettre en avant la notion de condominium sur le comté de Holstein.

La « terra » au-delà du Prince : la conscience juridique

12 Pour autant, cette connexion très forte entre dynastie Schauenburg et Holstein ne signifie pas que notre Presbyter fasse de la terra le simple théâtre de la geste du Prince. Le récit historique dénote également une conscience bien ancrée de la spécificité de la terra Holtzacie, à côté de ses dynastes. En tout premier lieu, il convient de se pencher sur l'arsenal de concepts géographiques, politiques et ethniques qui préside dans notre chronique à la définition de la terra. Ceux-ci en effet, pour reprendre les termes de Jean-Marie Moeglin, «ne peuvent être considérés comme de simples outils ; ils ont leur propre histoire et leur propre force. Les sens dont ils arrivent chargés de par leur histoire conditionnent à la fois leur réutilisation dans de nouveaux contextes et orientent cette réutilisation $»^{25}$.

Or l'histoire de ces concepts pour la "Nordalbingie " nous ramène encore une fois à l'œuvre fondatrice d'Helmold de Bosau, source de référence de notre auteur du $\mathrm{xv}^{\mathrm{e}}$ siècle. Les chapitres directement inspirés d'Helmold marquent des inflexions significatives : sans renier l'arsenal assez profus des désignations helmoldiennes, notre auteur effectue un tri raisonné et montre où vont ses préférences : terra, ainsi que la désignation par le nom propre seul, l'emportent très nettement sur d'autres notions. Les grandes victimes de ce tri sont d'une part la vieille nomenclature érudite, chargée de réminiscences carolingiennes sinon romaines, provincia/pagus, encore assez présente chez Helmold au dernier tiers du xII ${ }^{\mathrm{e}}$ siècle, et d'autre part le concept original et englobant de Nordalbingia - «terres au nord de l'Elbe ». Si patria n'est pas inconnu de notre auteur, le terme reste marginal, même si certaines de ses occurrences, en entretenant un certain flou autour de la notion, permettent habilement de faire du Holstein la patrie à la fois de la dynastie, de l'auteur et des Holsten en général ${ }^{26}$. Le terme dominateur est sans conteste terra tout au long de la chronique : il faut noter que ce terme peut être intégré à toute une série de composés lexicaux ("principes terre ", « comites terre Holtzacie »...), et n'est pas confiné à la terre de Holstein (il est également question plusieurs fois de la «terre des Dithmarscher» et de la «terre de Wagrie »). Une évolution parallèle est perceptible au niveau des noms propres géographiques: Holsacia, repris d'Helmold, supplante très largement Wagria, Stormaria et Ditmarsia, les autres composantes de la terre nordalbingienne qui sont fortement présentes chez Helmold de Bosau. Cela témoigne au premier chef d'un processus politique complexe, à la fois de différenciation et d'absorption. Wagrie et Stormarie ont en effet été intégrées au comté qu'il est alors de coutume d'appeler simplement de Holstein, alors que dans le même temps, la Ditmarsia, entre mer du Nord et Holstein, a connu son propre développement endogène et n'est que marginalement présente dans une chronique qui s'attache avant tout à un espace dynastique qui s'arrête aux limites du comté.

Mais si l'horizon du Chronicon, à l'âge d'or d'une historiographie dynastique, n'est plus une vaste Nordalbingia vide de sens politique et identitaire, il n'en reste pas moins que la terra n'est pas la simple projection géographique du dominium des Schauenburg. Dans l'esprit du Presbyter Bremensis, plusieurs terre coexistent dans ce dominium : dans son récit, les Frisons du Nord, «sujets » incontestables des Schauenburg, habitent une terra distincte, alors que le duché de Schleswig, s'il n'est certes pas désigné comme terra, a 
clairement un statut à part. Il semble en réalité que le critère essentiel pour la distinction d'une "terre" particulière soit de nature juridique. La conscience «territoriale» se fonde manifestement sur la reconnaissance de droits coutumiers différents. En contradiction avec la réalité contemporaine, mais aussi avec d'autres sources comme la Chronik der nordelbischen Sassen, un texte sans doute hambourgeois, composé entre 1459 et $1483^{27}$, le Presbyter ne dit rien de la diversité juridique à l'œuvre dans le comté de Holstein, qui renvoie à l'histoire de la colonisation des terres slaves ainsi que des zones insalubres ou côtières. Trois aspects méritent d'être évoqués à ce titre. Tout d'abord il faut noter que le patriotisme juridique prend dans le Chronicon la forme d'un saisissant travestissement de l'Histoire du comté. Ainsi l'époque de la mainmise danoise dans le Holstein au début du XIII ${ }^{\mathrm{e}}$ siècle, période que l'historiographie la plus récente s'accorde à considérer comme une étape décisive dans l'introduction de normes juridiques précises et notamment du droit féodal - futur fondement du Landrecht holsteinien de la fin du Moyen Âge - est dépeinte, par une sorte de déplacement du conflit, comme un moment d'affrontement acharné entre les Holsten défenseurs de leur droit régional et les officiers de la Couronne danoise désireux d'introduire leur propre droit écrit. Et le comte Adolphe IV de Schauenburg, par ailleurs l'un des artisans les plus résolus de la «normalisation juridique » et de la féodalisation au nord de l'Elbe, est célébré par le Presbyter comme le restaurateur de l'authentique droit ancestral holsteinien contre les innovations danoises, tel un nouveau Judas Macchabée: «Et tout comme Judas [Macchabée] avec l'aide divine, vainquit l'orgueilleux roi Antiochus, destructeur de la Loi juive, de la même façon le comte Adolphe épuisa Knut le roi du Danemark, qui s'efforçait d'opprimer par la force le droit holsteinien $»^{28}$.

Cela est d'autant plus frappant que, par ailleurs, le Presbyter paraît concevoir, avec une certaine fierté, le Holstein comme un avant-poste d'un droit supérieur (la féodalité) dans un monde où règnerait la barbarie juridique. Un passage révélateur dirigé contre les habitants de la Ditmarsia va dans ce sens : ces derniers y sont couverts de ridicule en raison de leur incapacité juridique à comprendre le sens féodal du verbe "concedere ", leur naïveté dans ce domaine les amenant à raisonner en termes éminemment prosaïques $^{29}$. Le troisième point de ce patriotisme juridique qu'il convient de relever ici touche au succès du droit lubeckois dans ces régions. Certes notre auteur n'ignore pas que la majorité des villes holsteiniennes ont adopté un droit directement dérivé de celui en usage dans la «capitale » de la Hanse ${ }^{30}$, mais il impute cette émulation à la seule volonté des comtes agissant uniquement pour le bien de la dynastie et, qui plus est, tend à nier discrètement la spécificité de ce droit urbain « en amont » : il affirme en effet avec aplomb que le droit lubeckois lui même n'est qu'un produit dérivé du Miroir des Saxons, ce grand recueil juridique du xiII ${ }^{\mathrm{e}}$ siècle qui fut la base de moult coutumiers du nord de l'Allemagne à la fin du Moyen Âge ${ }^{31}$.

La conscience de la spécificité holsteinienne ne souffre donc pas la contestation, mais il est bon d'ajouter néanmoins qu'elle ne devient jamais dans le Chronicon un étendard au nom duquel l'on se dresserait contre l'action de la dynastie comtale. Les récits guerriers qui abondent entrelacent sans cesse principes et Holtzati, et jamais leurs intérêts respectifs ne sont opposés ni même réellement distingués lors de la relation des conflits sans fin avec l'ennemi danois. Surtout, la noblesse territoriale ne se pose jamais ici en porte-parole de la « terre » contre la politique dynastique. À une époque où l'on assiste dans ces régions à la montée en puissance d'un « état » noble ${ }^{32}$, notre auteur répugne à 
en prendre acte. Certes apparaissent dans son récit quelques figures de conseillers importants autour des Princes, mais jamais la ridderscop n'intervient en temps que corps constitué, à la différence de ce que l'on observe dans la Chronik der nordelbischen Sassen évoquée plus haut, et les personnages ne s'imposent jamais dans le récit par leur rapport privilégié à la terra par-delà la fidélité à la dynastie comtale.

Le Holstein béni de Dieu

tout uniment se caractérisent également par une relation trè fortement affirmée au sacré, à une époque, ne l'oublions pas, où « la nation ne cherche pas encore à être une valeur laïque, mais au contraire à s'inscrire dans le sacré et à participer à son prestige $»^{33}$. Notons d'emblée que le simple fait d'opter pour le latin et pour le cadre de la Chronique Universelle dénote sans doute, à une époque où le vernaculaire gagne du terrain et où de nouvelles formes d'écriture de l'histoire détachées des anciens cadres eschatologiques tendent à s'imposer, un choix historiographique conscient : celui de replacer le destin de la terre de Holstein dans le vaste plan de Dieu et de l'Histoire du Monde. Le rappel insistant de bribes des vieilles conceptions qui liaient le Saint Empire à un arrière-plan apocalyptique et providentiel dans notre chronique va dans le même sens : le songe de Daniel parait l'épisode vétérotestamentaire qui intéresse le plus notre auteur ${ }^{34}$, et l'évocation du Christ n'est finalement guère autre chose ici que le prétexte à consigner la théorie médiévale des Deux Glaives temporel et spirituel ${ }^{35}$.

Il va sans dire cependant que le Holstein ne saurait être uniquement magnifié en tant que segment d'une Histoire providentielle du Monde ; l'Histoire nordalbingienne est aussi le lieu où se manifeste un lien particulier entre Dieu et une terre. La volonté divine, principe d'explication général, cède à l'occasion la place à la singulière alliance entre Dieu et le Holstein. Le Presbyter en fait parfois le «champion de la terre »« propugnator terre Holtzacie » - ou même le capitaine des châteaux comtaux menacés "Deus omnipotens castrum Gottorpe custodivit ${ }^{36}-$, sans parler des miracles qu'il accomplit au service de l'ost holsteinien, à qui il est par exemple donné de marcher sur l'eau pour détruire une flottille danoise ${ }^{37}$.

Plus encore que Dieu le Père, c'est la Vierge qui fait montre à l'égard du comté et de ses dynastes d'une sollicitude toute particulière. C'est au cri de Holstenland, vrouwe van hemmelrike («terre de Holstein, Vierge du Ciel») qu'un capitaine lance l'assaut contre l'assiégeant danois ${ }^{38}$. C'est encore à la Vierge que se recommande à l'aube d'une bataille décisive, par la récitation d'une hymne mariale, le comte Gérard le Grand (1304-1340), le grand artisan de l'expansion holsteinienne dans la première moitié du XIV siècle ${ }^{39}$. Cette insistance sur la figure mariale doit être interprétée, pour notre propos, à deux niveaux distincts. Il y a là, à n'en pas douter, un témoignage de l'extraordinaire essor du culte marial à la fin du Moyen Âge. Mais il est difficile de ne pas y voir aussi la marque d'un auteur dont on a vu les liens avec une nécropole comtale placée sous le patronage de la Vierge ${ }^{40}$, qui tout naturellement devient alors advocata et adjutrix ${ }^{41}$ de la terre de Holstein.

Dans une région au paysage intellectuel marqué par une historiographie de la Mission, d'Adam de Brême à Helmold de Bosau, il est intéressant de noter que le Presbyter paraît par ailleurs tendre à confondre les combats de la Maison de Schauenburg avec la cause de l'évangélisation - jamais vraiment achevée - en butte au paganisme. Remarquable à cet égard est la tentative discrète mais bien réelle de faire coïncider avènement des Schauenburg et authentique évangélisation, au rebours de la réalité 
historique. Elle se lit tout d'abord dans la minoration de la figure de saint Ansgar, premier apôtre du Nord germanique et slave par rapport à son successeur de xiie siècle, saint Vicelin. Au premier, malgré le témoignage d'Helmold ${ }^{42}$ auquel a accès notre auteur, le Presbyter n'accorde que la portion congrue dans son récit, à la différence de la Chronik der norbelbischen Sassen déjà évoquée. Vicelin en revanche retient l'attention admirative du Presbyter. Celui-ci avoue presque ses arrière-pensées lorsqu'il reprend spectaculairement une phrase d'Helmold presque mot pour mot pour décrire la situation à laquelle Vicelin fut confronté au nord de l'Elbe : « Et dans cette même région [sc. la terre de Holstein], bien que l'on réputât ses habitants chrétiens, le culte idolâtre et vain des bois et des sources était répandu $»^{43}$.

21 Or, la mission de Vicelin est quasi contemporaine de l'arrivée des Schauenburg et de l'inféodation d'Adolphe $\mathrm{I}^{\mathrm{er}}$. Et notre auteur ne se prive pas de mettre à profit cette concomitance pour faire en quelque sorte des premiers comtes Schauenburg les « coévangélisateurs » de la terra. Les fondations de monastères sous l'égide de saint Vicelin mettent en avant le rôle d'Adolphe $\mathrm{I}^{\text {er }}$, alors que le Presbyter, au contraire, omet soigneusement d'évoquer les épisodes de tensions entre le comte et le saint que l'on trouve chez Helmold, à propos notamment de la dotation de l'évêché nouvellement fondé d'Oldenburg dans l'est du Holstein. Ce gauchissement des rôles respectifs dans l'évangélisation des populations au nord de l'Elbe trouve son aboutissement dans une remarque incidente du Presbyter plus loin dans le récit, où il est question d'Adolphe IV : « Cet Adolphe régna sur le comté, comme de nombreux autres avant lui, également appelés Adolphe, nés de cette Maison, avaient régné sur la terre de Holstein, en princes très vertueux, depuis les débuts du christianisme $»^{44}$. Par la suite, cette confusion entre fonction guerrière et mission évangélisatrice ne se dément pas: les Danois, et les Dithmarscher, habituels rivaux des comtes de Holstein, se voient plus ou moins explicitement prêter des pratiques crypto-païennes.

L'alliance de Dieu, de la terra et de la dynastie est définitivement scellée dans le récit du Presbyter à travers la figure d'Adolphe IV, alias « Frère Adolphe ». Ce personnage peu banal est le vainqueur de Bornhöved en 1227 et le restaurateur des Schauenburg. En 1239 , au terme d'un engagement spirituel de plus en plus manifeste, il finit par entrer chez les Franciscains à Hambourg puis à Notre-Dame de Kiel. Il y résidera en odeur de sainteté jusqu'à sa mort en 1261. À l'instar de cette existence double, le portrait du Presbyter, qui s'étend sur plusieurs pages, est nettement bipartite. Adolphe IV est d'abord le champion de la terre de Holstein, le nouveau seigneur et prince qui chasse les usurpateurs à la solde du roi du Danemark. Puis Frère Adolphe éclipse Adolphe IV, et le Presbyter se délecte manifestement à relater différents épisodes légendaires de cette deuxième vie: rencontre avec saint François d'Assise en Italie, confrontation hautement symbolique entre le vieux frère franciscain déguenillé et ses fils et successeurs à cheval dans les rues de Kiel ${ }^{45}$. Si Henri de Fer (1340-vers 1384), le valeureux et turbulent comte de Holstein de la fin du XIv siècle, est par bien des aspects l'honneur chevaleresque de la dynastie, Frère Adolphe en incarne la piété insigne, et couronne l'image de l'alliance entre terre, dynastie et plan de Dieu.

23 Pour autant, le Presbyter paraît étrangement ignorer toute une série de faits mémorables de la vie franciscaine de Frère Adolphe, que d'autres historiens un peu antérieurs, tels Hermann de Lerbeck ou l'auteur anonyme de la Holsteinische Reimchronik (Chronique Rimée du Holstein), relèvent dans leur portrait du saint comte: sa participation à une «reise " en Livonie en 1238, tout comme la date exacte de son 
entrée dans les ordres. Mais c'est que nous avons affaire avec ces deux derniers auteurs à des Mendiants. Sans doute témoignent-ils à leur façon d'un phénomène bien décelable $a u \mathrm{xv}^{\mathrm{e}}$ siècle, à savoir l'essor d'une tradition de commémoration de la pia memoria d'Adolphe IV chez les Franciscains et les Dominicains, dans des établissements souvent fondés au XIII ${ }^{\mathrm{e}}$ siècle sous l'égide d'Adolphe IV lui-même. Dès 1340, un manuscrit de Greifswald le range parmi les saints franciscains de la province de Saxe même si aucune tentative de canonisation ne nous est connue, et l'existence d'un monument funéraire tardo-médiéval à sa mémoire ainsi que d'un opuscule d'un Franciscain hambourgeois intitulé « De l'illustre Adolphe comte de Holstein de l'ordre des Frères Mineurs à Kiel $»^{46}$ attestent du développement de ce programme commémoratif au cours $d u x^{e}$ siècle. Notre Presbyter, s'il ne se fait pas faute de rappeler le glorieux souvenir de Frère Adolphe, est incontestablement extérieur à un programme de célébration qui est en même temps, n'en doutons pas, une tentative d'affirmation d'un pôle dynastique indépendant, et dans une certaine mesure, rival d'Itzehoe. Peut-être faut-il voir là une autre raison qui pousse le Presbyter à entreprendre avec tant de vigueur la défense et illustration de la nécropole comtale d'Itzehoe en ces temps de crise dynastique.

La terre de Holstein face aux entités politiques de référence : Saxe et Empire

Une dernière dimension reste à explorer brièvement ici. À la différence de l'historiographie des plus grandes principautés de l'Empire, celle centrée sur des territoires plus modestes, qui ne disposent pas de la même marge de manœuvre ni du même horizon politique, doit articuler une identité régionale non seulement au passé immémorial mais également aux autres pouvoirs contemporains. Nous pouvons discerner ce processus à l'œuvre dans le Chronicon. Saisir l'identité historique du Holstein revient en effet immanquablement à se plonger dans un passé où celui-ci se trouvait dans l'ombre de deux entités politiques plus vastes : l'Imperium et la Saxonia.

Le premier de ces deux cadres nimbés de gloire, s'il est comme nous le suggérions plus haut un précieux instrument idéologique en ce qu'il replace l'Histoire du Holstein dans le cadre de l'Histoire Universelle, est clairement une réalité politique évanescente dans la Nordalbingie de 1448. Ainsi, si vingt-trois empereurs sont nommément cités dans la Chronique d'Helmold, de Charlemagne à Lothaire de Süpplinburg, les passages correspondants dans le Chronicon n'en évoquent que huit. Notre auteur semble en outre plus impressionné par la puissance du roi de France, «le plus noble et le plus riche des princes et rois du monde $»^{47}$ que par la vigueur de l'Empire germanique. Rien ne montre mieux la fonction adventice de la référence impériale que la présentation que fait le Presbyter du séjour de Charles IV à Lubeck en 1375. Après avoir commencé d'évoquer les hauts faits du belliqueux Henri de Fer, le Presbyter enchaîne alors comme suit : " Il se trouva alors que Charles IV, roi des Romains et empereur, rendit visite à la cité de Lubeck $»^{48}$. L'empereur tranche en fait un contentieux entre les Schauenburg et la cité de Hambourg au profit des comtes. La décision impériale n'est qu'une péripétie certes notable, mais péripétie malgré tout - de la longue geste du comte Henri de Fer. Les Princes de Holstein demeurent de bout en bout le fil directeur du récit.

Les choses sont différentes en ce qui concerne l'antique Saxonia. La référence saxonne, significativement, se trouve placée sous le signe de l'ambivalence. Le Presbyter nous livre, d'une part, un récit d'origine des Holsten qui est directement lié à l'origo Saxonum. La narration à ce point de son œuvre emprunte manifestement un vieux schéma d'ethnogenèse saxonne qui trouve sa source dans Widukind de Corvey ${ }^{49}$ repris par 
Frutolf de Michelsberg ${ }^{50}$. Néanmoins, notre auteur, qui dit avoir puisé "dans une chronique $\|^{51}$ ses informations, présente un récit singulier, dans lequel certains éléments originaux n'ont pas leur pendant ailleurs. Il affirme ainsi que les Saxons sont des compagnons de lutte d'Alexandre le Grand qui auraient migré par voie de mer vers l'embouchure de l'Elbe et les rivages de la Mer du Nord et de la Baltique : jusque-là, nous avons affaire à des éléments qui remontent à Widukind pour l'essentiel. Leur nom dériverait de leur courage de pierre au combat ${ }^{52}$, une affirmation qui n'est pas absolument neuve ${ }^{53}$, mais qui contredit néanmoins l'étymologie classique proposée par Widukind et les auteurs qui l'ont suivi. Quoi qu'il en soit de la source exacte du Presbyter pour ce passage, difficilement déterminable ${ }^{54}$, l'on peut noter que ce récit auréolé de la gloire saxonne est habilement adapté au cas des Holsten: ceux-ci ne seraient que "les plus nobles des Saxons" dont le navire se serait engagé dans l'estuaire de l'Elbe et de la Stör. Le Presbyter revient ensuite au schéma classique : il ne reste ensuite aux nouveaux venus, sous la conduite d'un jeune homme anonyme, qu'à expulser les "Thuringiens » qui occupaient la région, et n'avaient de cesse de harceler les Saxons. La parenté saxonne, qui permet de rattacher les Holsten aux grandes nationes germaniques du haut Moyen Âge, est donc ouvertement revendiquée.

Ce détournement de la gloire saxonne apparaît également au travers de l'« annexion » de la figure d'Henri le Lion. Le Presbyter, là encore, gomme soigneusement ce qui dans Helmold révèle trop crûment les frictions - incessantes - entre le Welf et le comte de Holstein Schauenburg. L'arrimage du Lion à la terre et la dynastie comtale va même plus loin. Le Presbyter prétend ainsi que l'attribution du prénom Henri à de nombreux membres du lignage Schauenburg se fait en mémoire du duc Henri le Lion et d'un autre "Henri de Thuringe » que le premier avait désigné comme tuteur du jeune Adolphe $\mathrm{III}^{55}$. Au détour d'une phrase, il honore du reste le même Henri le Lion du titre de " champion de la terre de Holstein ${ }^{56}$, un titre qu'il réserve par ailleurs au seul comte Nicolas, vaillant défenseur de l'intégrité du comté contre les menées danoises à la fin du XIV ${ }^{e}$ siècle, et frère du preux Henri de Fer.

D'autre part, en revanche, le Presbyter passe soigneusement sous silence la suzeraineté saxonne, bien réelle jusqu'à 1427, qui pèse sur le comté. L'antique ascendance saxonne va de pair ici avec un déni de suzeraineté à l'égard des ducs de Saxe-Lauenburg. Ceux-ci sont évoqués comme des princes territoriaux parmi d'autres, à l'instar d'un Éric IV (1368-1412) ou d'un Éric V (1412-1436), dont l'interventionnisme belliqueux au nord de l'Elbe s'explique pourtant en partie par la volonté de regagner une influence effective dans le comté. Notre auteur omet soigneusement la suzeraineté sur le comté de Holstein lorsqu'il décline la longue titulature des ducs : « duc de Saxe, maréchal [sc. de l'Empire], électeur d'Empire et seigneur de Lunebourg ${ }^{57}$. Cette relation ambiguë témoigne, sans doute, des difficultés d'une historiographie infra-princière, dont l'un des défis est à la fois d'asseoir l'autonomie de la terra et d'auréoler celle-ci de la gloire d'entités plus prestigieuses et mieux attestées pour les « Siècles Obscurs ».

La volonté d'écarter du tableau de l'histoire du Holstein toute marque d'influence d'entités politiques plus vastes va de pair dans le Chronicon avec l'affirmation de l'emprise comtale sur les villes, qui, elles aussi, en retour, cherchent à échapper à la tutelle féodale. Le récit de notre auteur est ici en réalité paradoxal. D'un côté, ses nombreuses mentions de l'immixtion des consuls de Hambourg et Lubeck dans les conflits des Schauenburg, au titre de médiateurs et de conciliateurs, portent témoignage de la montée en puissance des deux grandes cités hanséatiques sur la scène 
politique régionale ${ }^{58}$. L'enjeu que constitue une aide militaire hambourgeoise que les comtes ne sont plus en mesure d'exiger au titre du service d'ost transparait nettement dans le Presbyter Bremensis, alors que Lubeck est plus d'une fois stigmatisée comme l'alliée des Danois honnis. Mais, par ailleurs, certains passages attestent de la prégnance d'une certaine nostalgie de la «bonne ville » comtale, soumise et fidèle. À ce titre, la façon dont notre auteur rapporte les crises urbaines qui firent rage dans de nombreuses cités de la région au début $\mathrm{du}_{\mathrm{xv}} \mathrm{v}^{\mathrm{e}}$ siècle, ne laisse pas d'étonner. $\mathrm{Au}$ minimum, les troubles urbains sont l'occasion de réaffirmer haut et fort la fidélité des villes à la dynastie contre vents et marées (cas de Flensburg, alors qu'il s'agissait bien plus d'une lutte entre clans rivaux $)^{59}$. Le Presbyter va même jusqu'à métamorphoser un conflit aux ressorts proprement urbains en un affrontement passionnel pour ou contre le maintien de liens privilégiés entre la puissance comtale et les cités : c'est le cas pour Hambourg, où le commun s'en prend aux édiles en place qui répugnent à venir militairement au secours des Schauenburg ${ }^{60}$. Dans cette optique, les patriciats urbains en difficulté tendent donc à devenir, avec plus ou moins de netteté, les oppresseurs des bourgeoisies locales et les suppôts des ennemis jurés de la terre et des comtes, tout uniment (Hambourg, Lubeck) ${ }^{61}$.

L'identité holsteinienne s'exprime enfin au travers des considérations qui émaillent le texte au sujet des turbulents voisins occidentaux du comté, les paysans libres des Dithmarschen. Ceux-ci, vassaux théoriques de l'archevêque de Brême, forment une sorte de confédération de paroisses gouvernées par un système complexe d'essence clanique $^{62}$. Le Presbyter, qui paraît ignorer largement le détail du fonctionnement politique et institutionnel de cette "République paysanne ", n'a de cesse d'accuser ces voisins de tous les maux et de tous les crimes. Il semble que la croyance en la «barbarie » foncière de ces paysans fonctionne comme un principe d'explication bien commode d'un régime politique et judiciaire profondément original, fait à la fois de survivances du vieux droit clanique saxon et d'une organisation socio-politique adaptée aux conditions d'exploitation des terres de polder. Le jugement final du Presbyter, sans appel, est révélateur : «c'est avec difficulté que l'on pouvait obtenir d'eux que justice soit rendue. Ces Dithmarschen, vivant sans prince et sans "chef", font ce qu'ils veulent $»^{63}$. L'absence de prince - qui est en même temps un principe dans cette vision organiciste du pouvoir - est synonyme d'anarchie aux yeux de notre auteur, qui par là accrédite encore plus, a contrario, la cohérence de la trinité terra/princeps/gens à l'œuvre dans le Holstein.

Réception : une chronique dynastique détournée?

L'identité holsteinienne telle qu'elle se formulait au milieu du $\mathrm{Xv}^{\mathrm{e}}$ siècle apparait donc maintenant avec plus de netteté, dans ses diverses composantes. Encore faut-il, pour pouvoir juger de la "pertinence» et de la "représentativité » de ce discours identitaire, se pencher sur le devenir du Chronicon Holtzatiae. La postérité de l'œuvre du Presbyter Bremensis est en fait, de prime abord, placée sous le signe du paradoxe. Le Chronicon a effectivement connu une certaine diffusion à la fin $d u x^{e}$ siècle et tout au long du $\mathrm{xVI}^{\mathrm{e}}$ siècle, alors que la rupture dynastique qui, à notre sens, l'explique et sans aucun doute a motivé sa rédaction a eu lieu dès 1459 , et que Christian d'Oldenburg, succédant à Adolphe VIII de Schauenburg selon les vœux de ce dernier, confirme très rapidement les privilèges et prérogatives $d u$ «monastère dynastique » d'Itzehoe ${ }^{64}$. De ce texte " caduc ", nous avons gardé la trace de quinze manuscrits en tout et pour tout: cinq manuscrits latins et dix manuscrits allemands (en bas allemand). 
Ce n'est pas le lieu ici de discuter en détail les arguments paléographiques de Westphalen et Lappenberg. Contentons-nous de brosser un tableau rapide de la diffusion du Chronicon. Premier point notable : la diffusion de notre chronique semble ne commencer véritablement qu'à l'aube $d u x v I^{e}$ siècle. Des quinze manuscrits connus, un seul fut rédigé avec certitude au $\mathrm{XV}^{\mathrm{e}}$ siècle, contre sept copiés certainement ou très vraisemblablement entre 1500 et 1600 (quatre manuscrits latins et trois vernaculaires). Il ne faut pas oublier, par ailleurs, que l'on ne ressentit le besoin d'éditer le texte qu'en 1698. $\mathrm{Si}$, pour reprendre les catégories définies par Bernard Guenée ${ }^{65}$, le « succès » de l'œuvre fut bel et bien limité, il ne fut pas néanmoins négligeable, et, surtout, son "influence» fut loin d'être nulle. Tout au long du siècle, de nombreux érudits et chroniqueurs pillent en effet notre texte, sans toujours faire état de leur dette il est vrai, depuis le grand historien Albert Krantz jusqu'aux chroniqueurs dithmarschiens en passant par l'homme d'État et poète dans le goût humaniste que fut Henrik Rantzau. Par ailleurs, si l'on a de bonnes raisons de croire que le Chronicon n'a guère franchi l'Elbe vers le Sud, il a été lu dans le duché de Schleswig voire dans le royaume de Danemark, et dans les Dithmarschen, en dehors donc de la terra Holtzacie. Il ne s'est pas arrêté à la porte des villes, puisque l'on peut suivre sa trace à Hambourg comme à Lubeck, voire dans la cité épiscopale de Schleswig, au Xvi siècle.

Certes l'une des raisons de cette pérennité tient sans aucun doute à la relative pauvreté de l'historiographie holsteinienne médiévale, à "l'obscurité d'annales trop peu explicites ${ }^{66}$ pour reprendre les termes de Albert Krantz dans sa Saxonia, qui contraint les historiens à se contenter des rares textes existants. Mais est-ce tout ? C'est à ce point de l'analyse que la comparaison entre le texte latin et les versions basallemandes, qui paraissent avoir été toutes élaborées au $\mathrm{XVI}^{\mathrm{e}}$ siècle, peut nous prodiguer quelques lumières partielles sur la motivation des hommes qui ont sauvé de l'oubli cette œuvre à l'aube d'une époque nouvelle. Il semble en effet que les trois manuscrits allemands intéressants, qui, à la lumière de l'apparat critique de Westphalen ${ }^{67}$, semblent très proches, remontent tous les trois au xvi siècle, à la différence de ce que pensait Westphalen, suivi partiellement par Lappenberg dans sa préface à l'édition de 1862. L'un, celui de Copenhague, peut être daté de 1539, sur la foi d'un ex-libris incontestable. Les deux autres, dits «manuscrit de Held » et «manuscrit de Kirchhof » présentent tous les deux des altérations minimes qui font soupçonner une main " protestante ", et le manuscrit de Kirchhof inclut même une liste des "lignages éteints du Holstein ${ }^{68}$ qui fait état de familles nobiliaires qui apparaissent encore pour la majorité d'entre elles dans deux status nobilium de 1564 et de $1590^{69}$.

Les menus remaniements du texte latin attestent, globalement, de la persistance d'un schéma identitaire dont le Presbyter se faisait l'écho. Tout juste est-il intéressant de constater que les traducteurs du $\mathrm{XvI}^{\mathrm{e}}$ siècle, par les erreurs d'interprétation qu'ils commettent, trahissent une méconnaissance flagrante de la chronique d'Helmold, pourtant encore le texte de référence de notre auteur un siècle plus tôt. Le $\mathrm{Xv}^{\mathrm{e}}$ siècle finissant aurait-il été le chant du cygne de la tradition historiographique helmoldienne ? On peut se poser la question. Par ailleurs, la généalogie des comtes de Holstein qui clôt le manuscrit de Kirchhof permet de voir la fidélité au schéma de la continuité dynastique sans faille des Schauenburg : alors même que le copiste connaît et Albert Krantz et l'auteur anonyme de la Chronik der nordelbischen Sassen déjà mentionnée, deux auteurs qui ne font pas mystère de l'expulsion des comtes Schauenburg au début du XIII ${ }^{\mathrm{e}}$ siècle, il n'en fait nullement état dans sa généalogie il est 
vrai simplifiée et fautive ${ }^{70}$. Quelles sont les raisons essentielles de cet attachement ad litteram au texte du Presbyter, alors que la Maison d'oldenburg a remplacé les Schauenburg et que le mythe des origines saxonnes fait nettement place au mythe, plus conforme à l'esprit humaniste, des origines cimbres ${ }^{71}$ ?

C'est sans doute ailleurs, à notre avis, qu'il convient de chercher les raisons de l'attrait du Chronicon pour les lettrés du $\mathrm{XvI}^{\mathrm{e}}$ siècle. De la mort de Christian $\mathrm{I}^{\mathrm{er}} \mathrm{d}^{\prime}$ Oldenburg (1481) jusqu'au dernier tiers du siècle suivant, la noblesse holsteinienne, désormais solidement constituée en corps conscient de son rôle, n'a de cesse de lutter contre les tentatives toujours répétées des Oldenburg, parallèlement rois du Danemark, pour intégrer les deux duchés de Schleswig et de Holstein dans l'orbite danoise afin d'harmoniser leurs différents territoires. Les lignes de force du texte du Presbyter ne pouvaient de fait n'être que bienvenues dans ce contexte: image d'une terra sans suzerain, faiblesse de la logique strictement dynastique au nom de laquelle la Maison d'oldenburg prétend justement rogner les privilèges de la Ritterschaft holsteinienne, hostilité générale à l'égard du royaume du Danemark, insistance sur une identité propre de la terra Holtzacie...

Il ne s'agit là que d'une hypothèse, qu'il faut considérer comme telle. Néanmoins, de menus détails, insuffisamment remarqués jusqu'à présent, convergent pour trahir la réappropriation de cette chronique proprement "dynastique » et "princière " par de grandes familles nobles soucieuses d'affirmer leur droit à régir de facto le duché de Holstein $^{72}$. Il est difficile de croire, par exemple, eu égard au contexte que nous venons d'évoquer, que la dilution de l'expression "duc de la terre de Holstein » appliquée à Henri le Lion, qui, dans les versions bas-allemandes, devient simplement « champion et protecteur (avoué ?) de la terre de Holstein $»^{73}$ est accidentelle. On pourrait émettre les mêmes doutes fondés au sujet de la modification du passage concernant la frontière ancestrale entre royaume de Danemark et comté de Holstein sur la rivière Eider : le cuius limes fuit in Egdora s'est changé insidieusement en "dess ende iss an der Eyder $»^{74}$ ( dont la limite se situe - et non se situait - sur l'Eider »).

Faut-il enfin imputer au seul hasard le fait que ce sont précisément certains passages évoquant les ancêtres des plus puissantes familles du Holstein au $\mathrm{xVI}^{\mathrm{e}}$ siècle qui subissent le toilettage le plus significatif? À cet égard, le récit du désastre de la Suederhamme en 1404 - une des nombreuses tentatives avortées des comtes Schauenburg pour réduire les turbulents paysans des Dithmarschen - s'écarte assez sensiblement dans nos versions basallemandes du texte latin. Par ailleurs plutôt flatteur à l'égard des Reventlow, Rantzau et autres Ahlefeldt - les lignages qui, quelques décennies après la rupture dynastique de 1460, occupent clairement le haut du pavé -, notre texte incrimine ici deux frères Ahlefeldt, qui, par leur incurie, auraient été responsables de la défaite inattendue contre l'infanterie dithmarschienne ${ }^{75}$. La version allemande telle qu'on la trouve imprimée chez Westphalen saute purement et simplement cette longue phrase manifestement embarrassante, cas unique dans toute la traduction ${ }^{76}$. De là à penser que nos versions allemandes dérivent toutes d'une traduction primitivement réalisée à l'initiative de la famille Ahlefeldt, il n'y a qu'un pas. Et n'oublions pas que l'auteur du manuscrit de Kirchhof, à un moment difficilement identifiable $\mathrm{du} \mathrm{XVI}^{\mathrm{e}}$ siècle, choisit d'ajouter à la chronique une courte généalogie des Schauenburg et, dans la continuité, une liste de noms et d'armoiries de lignages nobiliaires éteints. 
Au terme de ce parcours, il nous semble que quelques traits peuvent être dégagés avec une certaine confiance. Ce qui domine dans le Chronicon Holtzatiae est incontestablement l'idée d'une union consubstantielle entre une terra à l'identité singulière et une dynastie de principes profondément ancrés dans ce lieu. Rédigé dans un but bien précis, le texte du Presbyter n'en reflète pas moins des structures mentales qui caractérisent le discours de l'identité territoriale dans le Saint Empire à la fin du Moyen Âge. Il nous met en présence, en outre, d'autres traits récurrents de la formulation de l'identité régionale du temps. Le langage du sacré, sous la forme de l'insigne protection divine, en est une clef de voûte. Le droit joue manifestement un rôle considérable dans la genèse de ces identités régionales, même si la coutume n'est jamais ici, dans ce texte si favorable aux intérêts dynastiques, brandie contre la légitimité princière. Mais ce sont aussi les défis d'une historiographie que l'on serait tenté d'appeler "infra-princière » (au sens des Princes d'Empire) qui transparaissent ici : comment à la fois annexer la gloire d'origines saxonnes tout en s'en tenant à une vision du Holstein « autonome " ? C'est là sans doute le dilemme de "l'historiographie du pauvre », et en cela la conscience de soi des comtes de Holstein reflétée par le Cronicon se distingue nettement des discours élaborés dans l'entourage des grands lignages de l'Empire, que des travaux récents nous permettent de cerner avec une certaine précision ${ }^{77}$. Ce texte obscur montre également qu'un discours identitaire s'étudie nécessairement dans la longue durée, parallèlement à sa postérité même. Les vicissitudes du devenir d'un tel discours sont parfois surprenantes, tout comme sa capacité à être récupéré dans un contexte radicalement nouveau : n'y a-t-il pas ici de bonnes raisons de croire que cet éloge de la dynastie Schauenburg et du «bon duc Adolphe» en particulier fut, quelque cent ans plus tard, l'une des armes de la Ritterschaft holsteinienne, que le Presbyter s'applique manifestement à passer sous silence comme corps, contre les excès du duc Oldenburg? «La citation a un nez de cire » disait le subtil théologien scolastique Alain de Lille. Gageons que c'est aussi parfois le cas de chroniques entières.

\section{NOTES}

1. B. Guenée, Histoire et Culture historique dans l'Occident médiéval, Paris, 1980, p. 64.

2. L'historiographie du Moyen Âge tardif, longtemps parent pauvre de la recherche allemande sur l'historiographie médiévale, a suscité un intérêt croissant depuis une vingtaine d'années, et la bibliographie sur le sujet s'est considérablement accrue en même temps que les problématiques se renouvelaient. On peut citer ici quelques grandes études ou travaux qui ont fait date : H. PATZE (éd.), Geschichtsschreibung und Geschichtsbewusstsein im späten Mittelalter, Sigmaringen, 1987 (Vorträge und Forschungen 31) ; R. SPRANDEL, Chronisten als Zeitzeugen, Cologne/Weimar/Vienne, 1994 ; P. WUNDERLI (éd.), Herkunft und Ursprung, Historische und mythische Formen der Legitimation, Sigmaringen, 1994. Voir aussi en dernier lieu G. GLEBA (éd.), Instrumentalisierung von Historiographie im Mittelalter, 2000 (= Das Mittelalter 2000, 2). 
3. E. J. von WeStPhALEN (éd.), Monumenta Inedita rerum Germanicarum praecipue Cimbricarum et Megapolensium, III vol., Leipzig, 1739-1745.

4. Chronicon Holtzatiae auctore Presbytero Bremensi, éd. J. M. LAPPENBERG (Quellensammlung der Schleswig-Holstein-Lauenburgischen Gesellschaft für vaterländische Geschichte), Kiel 1862. (désormais cité Chr. Holtz.)

5. M. Olivier, « Nemet iuwe Saxen! » : l'identité régionale dans le comté de Holstein au $X V^{\mathrm{e}}$ siècle à partir du « Chronicon Holtzatiae Auctore Presbytero Bremensi », sous la dir. de J.-M. Moeglin, Université de Paris XII, juin 2000.

6. Chr. Holtz., ch. 15, p. 30, 1. 12.

7. Schleswig-Holsteinische Regesten und Urkunden (Kloster Itzehoe 1256-1564), bd 8, bearb. vON H. H. HENNINGS, hg. vom Schleswig-Holsteinischen Landesarchiv, Neumünster, 1993, doc. 102 (1381), 144 (1410) et 162 (1421) (nous citons désormais SHRU), ainsi que Acta Pontificum Danica, vol. III, A. Krarup, J. Lindbaek (éd.), Copenhague, 1908, n. 1658 (juin 1432) sont ici les témoignages décisifs.

8. Il est difficile de se faire une idée exacte du nombre de clercs liés de par leurs fonctions aux moniales d'Itzehoe, sans parler même des vicaires évoqués ci-dessus. Une rapide enquête dans les SHRU sur les actes de ou concernant l'abbaye d'Itzehoe contemporains de notre auteur a permis d'en découvrir une dizaine. Cf. SHRU 4, 1324, 1399 ; SHRU 4-2, 120 ; SHRU 8, 139, 198... Ce relevé ne prétend pas ici à l'exhaustivité.

9. Sur la situation complexe du Schleswig entre ambitions comtales et suzeraineté danoise voir E. Hoffmann (éd.), Geschichte Schleswig-Holstein bd 4-2 : Spätmittelalter und Reformationszeit, Neumünster, 1990, p. 161-242.

10. Chr. Holtz., ch. 15, p. 30, 1. 8-11: « ad complementum cronice quam pie recordacionis frater Helmoldus [...] de Holzacorum principibus et vicinis eorum fideliter composuerat ».

11. J.-M. Moeglin, Les Ancêtres du Prince - propagande politique et construction d'une histoire nationale en Bavière au Moyen Âge (1180-1500), Genève, 1985 ;

également Id., « Dynastisches Bewusstsein und Geschichtsschreibung. Zum

Selbstverständnis der Wittelsbacher, Habsburger und Hohenzollern im Spätmittelalter », Historische Zeitschrift, 256, 1993, p. 593-635.

12. Chr. Holtz., ch. 9, p. 18 : « primus de regno et domo Francie... ».

13. Ibid., ch. 9 , p. 19 : « quando de domo Brunswicensi duces Saxoniam habebant... ».

14. Voir infra, p. 111.

15. Cf. SHRU bd. 10 passim, not. doc. 110 (1430).

16. Chr. Holtz., ch. 44, p. 143 : contra principes Sleswicenses et Holtzacie comites, non nominando eos (...) in literis duces sed comites.

17. H. de Lerbeck, Chronicon Comitum Schauenburgensium, éd. H. Meibom, Francfort/ Main, 1620. L'auteur, un Dominicain de Minden, a rédigé son texte vers 1410. Adolphe de Schauenburg, qui reçoit en fief le comté de Holstein en 1111, est le premier membre attesté avec certitude de ce lignage. Il était originaire de la moyenne vallée de la Weser (région de Rinteln) mais on ne sait rien de sûr au sujet des ancêtres de ce personnage dans la région. Cf. W. LAMMERS, Geschichte Schleswig-Holsteins bd 4-1 : das Hochmittelalter bis zur Schlacht von Bornhöved, Neumünster, 1981, p. 229-232.

18. Chr. Holtz., p. 27 ; voir aussi Holsteinische Reimchronik (anonyme), éd. L. WEILAND, MGH, Deutsche Kroniken II, 1877, p. 622, vs 354-359.

19. Chr. Holtz., ch. 12 , p. 25, 1. 4-10. 
20. Rimbertus, Vita Anskarii, éd. G. Waitz, mgh, Scr. Rer. Ger. in us. schol. 55, 1884 ; il s'agit d'un texte rédigé très vraisemblablement dans le dernier tiers du ix ${ }^{\mathrm{e}}$ siècle, peu après la mort de saint Ansgar en 865.

21. Chr. Holtz., p. 46, 1. 6 ; ch. 18, p. 48, 1. 5. La consultation des actes comtaux des xiii ${ }^{\mathrm{e}}$ et xiv ${ }^{\mathrm{e}}$ siècles montre à l'évidence qu'aucun des Teilgrafen n'a pourtant jamais intégré la Wagrie dans sa titulature. Cf. SHRU bd 2 et 3 passim.

22. Voir Chr. Holtz., ch. 18, p. 49, 1. 1-3 et pour un récit pro-Pinneberg radicalement différent Hermann de Lerbeck, op. cit., p. 39.

23. Chr. Holtz., ch. 18 , p. 49 ; ch. 20 , p. 61 . Là encore le Presbyter déforme la titulature authentique des comtes Schauenburg-Pinneberg, qui ne renoncent quasiment jamais au titre de « comte de Holstein ». Cf. H.-G. RISCH, Die Grafschaft Holstein-Pinneberg von ihren Anfängen bis zum Jahre 1640, Dissert. dactylo., Hambourg, 1986, passim.

24. Ibid., ch. 27, p. 94, 1. 9-12 d'une part, et SHRU bd 6, doc. 875 (13 avril 1390) d'autre part.

25. J.-M. Moeglin, « Nation et Nationalisme du Moyen Âge à l'époque moderne ", Revue Historique, 611, 1999, p. 540-553. À propos de la notion de patria dans le Saint Empire, voir l'article de P. Monnet, « La patria médiévale vue d'Allemagne, entre construction impériale et identités régionales » Le Moyen Âge, 107, 01/2001, p. 71-99.

26. Voir Chr. Holtz., ch. 16, p. 38 ; ch. 22, p. 70.

27. Chronik der nordelbischen Sassen, éd. J. M. LAPPENBERG (Quellensammlung der Schleswig-Holstein-Lauenburgischen Gesellschaft für vaterländische Geschichte), Kiel, 1865.

28. Chr. Holtz., ch. 16, p. 39 : « Nam ut Judas, divino fultus subsidio, superbum regem Anthiochum, legem Judaicam destruentem, devicit, ita et hic comes Adolphus Kanutum, regem Dacie, ius Holtzatense vi opprimere nitentem, enervavit ».

29. Ibid., ch. 43 , p. 142.

30. Ibid., ch. 17, p. 43 : « ...libertasse a iure Holtzatorum et favisse eis [i.e aliis opidanis per terram Holtzacie] ius lubicense eo ut eorum cicius muniretur propter libertatem fori ».

31. Ibid., ch. 15, p. 35 : «Dicte civitatis incolis ius municipale a speculo Saxonum extractum...».

32. I.-M. PETERS, « Der Ripener Vertrag von 1460 und die Anfänge der

Standesverfassung in Schleswig-Holstein », Blätter für deutsche Landesgeschichte, 109, 1973 et 111, 1975 ; l'auteur fait le point sur cette question de la genèse d'une Ritterschaft entre 1390 et 1460.

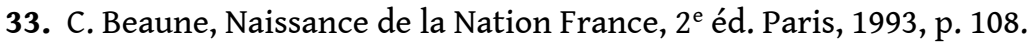

34. Chr. Holtz., ch. 5 , p. 7.

35. Ibid., ch. 6, p. 8-9.

36. Ibid., ch. 37, p. 125.

37. Ibid., ch. 36, p. 119.

38. Ibid., ch. 26, p. 91.

39. Ibid., ch. 19, p. 54 : « Nos hac die tibi congregatos serva virgo, in lucem mundi, qua... ».

40. Il semble que l'église abbatiale et paroissiale d'Itzehoe, vouée à l'origine à saint Laurent, un saint ottonien, soit à partir de la fin du xiv ${ }^{\mathrm{e}}$ siècle placée sous un double patronage dans lequel la Vierge occupe une position de plus en plus importante. Cf. E. IMBERGER, « Itzehoe im Spätmittelalter », dans J. IBS (dir.), Itzehoe-Geschichte einer Stadt in Schleswig-Holstein, Itzehoe, 1988, p. 39. 
41. Chr. Holtz., ch. 19, p. 55.

42. H. De Bosau, Chronica Slavorum, éd. B. SCHMEIDLER, MGH, Scr. Rer. Ger. ad us. schol.

32, 1932, I, 4.

43. Chr. Holtz., ch. 13, p. 26 : «Et ibidem quamvis christiani esse dicebantur, tamen lucorum et fontium error ac ydololatria (sic !) multiplex habebatur ».

44. Ibid., ch. 27, p. 93 : « Hic Adolphus prout etiam plures ante eum de tali domo nati sunt, eciam nominati Adolphus, rexerunt terram Holtzacie in magna virtute ab initio christianitatis ». C'est nous qui soulignons. Le latin du Presbyter est ici quelque peu hasardeux mais les traductions allemandes du Presbyter comprennent unanimement «depuis que la région est devenue chrétienne », ce qui va dans le sens de notre interprétation.

45. Ibid., ch. 17, p. 42-43.

46. De Inclito Adolpho comite Holtzacie ordine minorum in Kyl, éd. N. BEECK, Quellensammlung für Schleswig-Holsteinisch-Lauenburgische Geschichte vol. IV, Kiel, 1875, p. 205-227.

47. Chr. Holtz., ch. 24, p. 77.

48. Chr. Holtz., ch. 25, p. 82 : «Illis diebus contingebat, quod Carolus quartus, Romanorum rex et imperator, visitavit civitatem Lubeke... ».

49. Widukind de CoRvey, Res Gestae Saxonicae, éd. P. Hirsch, E. LoHMAnN, MGH Scr. Rer. Germ in us. scol. $5^{\mathrm{e}}$ éd., 1935, I, 1-9.

50. Voir Frutolf de Michelsberg, Chronicon, dans E. d'Aura, Chronica, éd. G. Waitz, mgh ss 7,1844$)$.

51. Chr. Holtz., ch. 8, 1. 8-9 : « Saxones vnde dicuntur uenisse, repperi in quadam chronica... ».

52. D'où leur nom originel de « Gens Petrita » qu'Alexandre le Grand aurait changé en "Saxons », de " saxum » = rocher, pierre.

53. Voir par exemple déjà Alcuin, De pontificibus et sanctis ecclesiae Eboracensis, PL. 101, col. 815 (vs 47) : « Duritiam propter dicti cognomine Saxi... »

54. Le Presbyter n'est certes pas le premier auteur nordalbingien à reprendre à son compte la théorie de l'origine " grecque » des Saxons telle que Widukind en avait fixé les linéaments. Albert de Stade, soit l'annaliste des Annales Stadenses, intègre ce récit sub anno 917. Cette notice se retrouve quasiment mot pour mot dans un appendice « de adventu Saxonum » d'un manuscrit d'un autre chroniqueur régional, Arnold de Lubeck (manuscrit dit « de Gottorp » par les éditeurs). Mais outre que le Presbyter ne connaît manifestement aucun de ces deux derniers auteurs, son récit ne coïncide pas vraiment avec celui d'Albert de Stade, même si l'on y retrouve quelques éléments secondaires à connotation « régionale » que l'Annaliste de Stade, est par ailleurs, à notre connaisance, le premier à rapporter. Cf. Annales Stadenses, éd. J. M. Lappenberg, mgh ss xvi, 1858, p. 311. Pour le rapprochement origo Holtzatorum/origo Saxonum voir également la glose du Miroir aux Saxons : Sachsenspiegel, éd. K. A. Eckardt, mgh Fontes Iuris Germanici Antiqui n.s, t. 1, 1933, III, 2-3, p. 133.

55. Chr. Holtz., ch. 15, p. 31.

56. Ibid., ch. 16, p. 36.

57. Ibid., ch. 27, p. 93 : « dux Saxonie, marschalcus, elector imperii et dominus Luneborgensis ".

58. Ibid., ch. 29, p. 101 ; ch. 31 , p. 105 ; ch. 40, p. 133.

59. Ibid., ch. 34, p. 114.

60. Ibid., ch. 37, p. 122-124. 
61. Voir, pour resituer le discours urbain du Presbyter dans un contexte plus large, A. Dirsch-Weigand, Stadt und Fürst in der Chronistik des Spätmittelalters, Cologne/ Vienne, 1991. Voir également sur les conflits urbains dans le monde hanséatique W. Ehbrecht, Konsens und Konflikt, Cologne/Weimar/Vienne, 2001 (= Städteforschung $\mathrm{A} / 56)$.

62. Sur les Dithmarschen au Moyen Âge et leur histoire singulière, voir H. Stoob, Geschichte Dithmarschens im Regentenzeitalter, Heide, 1959 ; aussi W. L. Urban, Dithmarschen. A Medieval Peasant Republic, New York, 1991.

63. Chr. Holtz., ch. 28, p. 96 : « ... cum difficultate ius ab eis haberi potest. Hii Ditmartici, sine principe et capite viventes, faciunt que volunt $»$.

64. La charte de confirmatio se trouve dans SHRU bd 8, doc. 235 (10 décembre 1460).

65. B. GUENÉE, op. cit., p. 257.

66. A. KRANTZ, Saxonia, dans : Chronica regnorum aquilonarium Daniae, Sueciae et Norwagiae, éd. A. Wechelus, Francfort/Main, 1575, vol. I, livre VIII, ch. 39.

67. Il convient en effet de préciser que deux de ces trois manuscrits allemands - celui dit « de Held » et celui dit « de Kirchhof » selon la nomenclature de Lappenberg - ont disparu entre l'époque de Westphalen et le moment où celui-ci prépare son édition en 1862.

68. E. J. VON WESTPHALEN, op. cit., t. III, col. 183-184.

69. Ibid., t. I, p. 5 et appendices (doc. C).

70. Ibid., t. III, col. 183-184.

71. C. Degn, Schleswig-Holstein, eine Landesgeschichte, Neumünster, 1994 not. p. 22-23 pour une description rapide de l'essor de cette cimbrophilie chez les auteurs du Nord de l'Allemagne.

72. Le comté de Holstein devient un duché en 1474.

73. E. J. VON WESTPHALEN, op. cit., t. III, col. 41/42.

74. Ibid., t. III, col. 47/48.

75. Chr. Holtz., ch. 31, p. 107.

76. E. J. von WeSTPHALEN, op. cit., t. III, col. 165/166.

77. Nous pensons ici aux différents travaux de J.-M. Moeglin sur les Hohenzollern, les landgraves de Thuringe, les ducs Wittelsbach de Bavière. Voir entre autres : « Dynasties princières allemandes et notion de Maison à la fin du Moyen Âge », dans Les Princes et le Pouvoir au Moyen Âge, $23^{\mathrm{e}}$ Congrès de la Société des Historiens Médiévistes, Brest, 1992, Paris, 1993, p. 137-154 ; ID., « Zur Entwicklung dynastischen Bewusstseins der Fürsten im Reich vom 13. zum 15. Jahrhundert », dans B. Schneidmüller (éd.), Die Welfen und ihr Braunschweiger Hof im hohen Mittelalter, Wiesbaden, 1995, p. 523-540 ; Id., " Sentiment d'identité régionale et historiographie en Thuringe à la fin du Moyen Âge », dans J.-M. Moeglin et R. Babel (éd.), Identités régionales et conscience nationale en France et en Allemagne du Moyen Âge à l'époque moderne, Sigmaringen, 1997, p. 325-363. 


\section{RÉSUMÉS}

L'auteur essaie de brosser le tableau de l'identité régionale dans le comté de Holstein au $\mathrm{Xv}^{\mathrm{e}}$ siècle, à partir d'une source principale, la chronique dite « du Presbyter Bremensis ", une œuvre latine rédigée en 1448. Le «Chronicon Holtzatiae » s'inscrit tout d'abord, sur le court terme, dans une perspective de crise dynastique imminente, et paraît très nettement appuyer par le recours à l'histoire les visées du dernier comte de Holstein Schauenburg Adolphe VIII, soucieux de barrer la route à ses cousins Pinneberg pour la succession comtale. Mais au-delà de ce contexte immédiat, un examen attentif permet d'observer les mécanismes du discours historique dans un texte d'orientation nettement princière et dynastique: si la «terre» ne se confond pas totalement avec la dynastie régnante, la plume du chroniqueur consacre néanmoins le lien consubstantiel entre le Holstein et ses comtes, même si, paradoxalement, il semble que le texte du Presbyter Bremensis ait surtout connu un succès tardif au xvI ${ }^{\mathrm{e}}$ siècle aux mains d'une noblesse territoriale soucieuse de défendre l'identité propre du Holstein dans la nouvelle donne politique que constitue l'union dynastique avec la couronne danoise.

Prince and History in the Mirror of the Chronicon Holtzatiae Auctore Presbytero Bremensi. This article deals with the issue of "regional» identity within the boundaries of a northern German principality in the late Middle Ages : the county of Holstein. The author, who draws upon his own MPhil dissertation, focuses himself on a quite ignored Latin chronicle from 1448, the so-called "Chronicon Holtzatiae» by an anonymous «Presbyter Bremensis ». He shows that first and foremost the chronicler, under the guise of history writing, buttresses the ambitions of the old and childless count of Holstein Adolf VIII of Schauenburg relating to the foreseeable « succession crisis » by excluding from the landscape of Holstein history throughout the centuries serious pretendants, i.e. the Schauenburg-Pinneberg. But, from a wider point of view, the «Presbyter Bremensis " also sketches a picture of "regional identity " which is typical of late medieval German dynastic historiography : the bond uniting the "terra " and the counts is depicted as a consubstantial one, constitutive of the very existence of the former, whereas other elements such as the regional law merely play a subordinate role.

\section{INDEX}

Mots-clés : Schleswig et Holstein, historiographie au bas Moyen Âge, dynastie et conscience identitaire, droit et identité régionale

Keywords : County of Holstein/Duchy of Schleswig, late medieval historiography, dynastic chronicles, law and « regional » identity

\section{AUTEUR}

\section{MATHIEU OLIVIER}

Université de Paris XII-Val de Marne, Département d'Histoire, Faculté des Lettres et Sciences Humaines, 61, avenue du général de Gaulle, F-94010 Créteil Cedex 